\title{
Desigualdades geográficas na implantação do Programa Mais Médicos em um estado brasileiro
}

\section{Geographical inequalities in the implementation of the More Doctors Program in a Brazilian state \\ Desigualdades geográficas en la implementación del Programa Más Médicos (Programa Mais Médicos) en un estado brasileño}

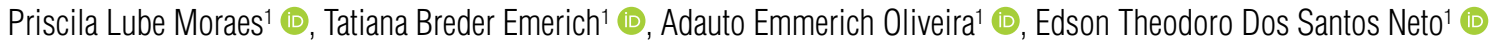

1Universidade Federal do Espírito Santo (IFES), Vitória, ES, Brasil

\section{Resumo}

Introdução: O Programa Mais Médicos (PMM) surge da necessidade de efetivar o direito universal de acesso ao Sistema Único de Saúde (SUS) e de tornar mais equitativa a assistência médica no Brasil. Objetivo: O estudo analisou o cenário de implantação do Programa Mais Médicos (PMM) nos 78 municípios do estado do Espírito Santo (ES) no primeiro ciclo do programa, de 2013 a 2016. Métodos: Trata-se de um estudo quantitativo, realizado com base em dados secundários coletados da Secretaria de Estado da Saúde; do Cadastro Nacional de Estabelecimentos de Saúde; do Instituto Brasileiro de Geografia e Estatística; e do Instituto Jones dos Santos Neves. Os municípios do Espírito Santo foram agrupados por portes populacionais, sendo a unidade de análise correspondente ao agregado populacional e ao espaço geográfico de cada município. Os dados foram analisados por meio de estatística descritiva e inferencial. Resultados: Os resultados deste estudo demonstraram que a implantação do PMM no Espírito Santo contribuiu para o fortalecimento da atenção primária, proporcionando o aumento e a fixação dos profissionais médicos em municípios tanto de pequeno quanto de maior porte populacional. No entanto, também se evidenciou que desigualdades geográficas verticais permaneceram durante a implantação do PMM entre municípios de porte populacional diferente. Conclusões: Ressalta-se a necessidade da gestão dos serviços da atenção primária na busca por um Sistema Único de Saúde resolutivo e equânime, independentemente do porte populacional.

Palavras-chave: Atenção primária à saúde. Sistema único de saúde. Acesso aos serviços de saúde.

Como citar: Moraes PL, Emerich TB, Oliveira AE, Santos Neto ET. Desigualdades geográficas na implantação do Programa Mais Médicos em um estado brasileiro. Rev Bras Med Fam Comunidade. 2021;16(43):2765. https://doi. org/10.5712/rbmfc16(43)2765
Autor correspondente:

Priscila Lube Moraes

E-mail: prilube @ ibest.com.br

Fonte de financiamento:

não se aplica.

Parecer CEP:

CAAE 58948516.5.0000.5060

Procedência:

não encomendado.

Avaliação por pares:

externa.

Recebido em: 26/10/2020

Aprovado em: 15/08/2021

Editor Associado:

Yuji Magalhães Ikuta 


\begin{abstract}
Introduction: The More Doctors Program (Programa Mais Médicos - PMM) arises from the need to enforce the universal right of access to the Brazilian public health system (Sistema Único de Saúde - SUS) and to make health care more equitable in Brazil. Objective: The study analyzed the first cycle (2013-2016) of PMM implementation in 78 municipalities of the state of Espírito Santo. Methods: This is a quantitative study based on secondary data collected from the State Health Secretariat, the National Record of Health Facilities, the Brazilian Institute of Geography and Statistics, and the Jones dos Santos Neves Institute. Municipalities of Espírito Santo were grouped by population size, with the unit of analysis corresponding to the aggregate population and geographic space of each municipality. Data were analyzed using descriptive and inferential statistics. Results: The results of this study showed that the PMM implementation in Espírito Santo contributed to strengthening primary care, increasing the number of physicians and establishing them in both smaller and larger cities. However, they also indicated that vertical geographical inequalities persisted during PMM implementation in municipalities of different population sizes. Conclusions: We emphasize the need for primary care management in the pursuit of an effective and equitable public health system, regardless of population size.
\end{abstract}

Keywords: Primary health care; Unified Health System; Health services accessibility.

\title{
Resumen
}

Introducción: El Programa Más Médicos (PMM) surge de la necesidad de hacer efectivo el derecho universal de acceso al Sistema Único de Salud (SUS) y de tornar más equitativa la atención médica en Brasil. Objetivo: El estudio analizó el escenario de implantación del Programa Más Médicos (PMM) en los 78 municipios del estado de Espírito Santo (ES) en la primera etapa del programa, de 2013 a 2016. Métodos: Se trata de un estudio cuantitativo, basado en datos secundarios recogidos de la Secretaría de Salud del Estado; del Registro Nacional de Establecimientos de Salud; del Instituto Brasileño de Geografía y Estadística; y del Instituto Jones dos Santos Neves. Los municipios de Espírito Santo fueron agrupados por tamaño de la población, siendo la unidad de análisis correspondiente el agregado de la población y el espacio geográfico de cada municipio. Los datos fueron analizados por medio de estadística descriptiva y deductiva. Resultados: Los resultados de este estudio mostraron que la implantación del PMM en Espírito Santo contribuyó con el fortalecimiento de la atención primaria, proporcionando un aumento y el establecimiento de los profesionales médicos, en municipios tanto de pequeño como de mayor porte poblacional. Sin embargo, también se evidenció que permanecieron desigualdades geográficas verticales durante la implantación del PMM entre municipios de porte poblacional diferente. Conclusiones: Se destaca la necesidad de administrar los servicios de atención primaria en la búsqueda por un Sistema Único de Salud resolutivo y ecuánime, independiente del tamaño de la población.

Palabras-clave: Atención primaria de salud. Sistema único de salud. Acceso a los servicios de salud.

\section{INTRODUÇÃO}

O Programa Mais Médicos (PMM) foi criado com as promessas de efetivar a garantia do acesso universal ao Sistema Único de Saúde (SUS) e de tornar mais equitativa a assistência médica no Brasil, objetivos essenciais na determinação social e no direito constitucional à saúde. ${ }^{1}$ No entanto, não se pode negar que os indicadores sociais preexistentes impactam as políticas públicas, além de serem agentes norteadores das desigualdades de um país. ${ }^{2} \mathrm{~A}$ pobreza, as condições precárias de moradia e as condições insalubres de trabalho interferem negativamente na determinação da saúde individual e coletiva. ${ }^{3}$ Dessa forma, as desigualdades sociais determinam o processo saúde-doença, ${ }^{4}$ traduzindo-se em desigualdades em saúde. Além disso, o contexto geopolítico exerce influência direta nesse processo, estabelecendo desigualdades entre regiões, estados e municípios. ${ }^{5}$

A partir dos anos 1980, a descentralização político-administrativa do Brasil associou-se com a luta pela redemocratização. Nesse período, aconteceram as eleições para estados e municípios antes da eleição direta para a presidência da República, o que intensificou a emancipação política. Isso num momento em que os governos subnacionais assumiram maiores responsabilidades e ocorreram incentivos e transferências livres de recursos fiscais da União, ${ }^{6}$ estabelecidos sem critérios, contribuindo para as desigualdades regionais ${ }^{7}$ que impactam as desigualdades sociais e a utilização dos serviços de saúde. ${ }^{8}$ 
A fim de dirimir as desigualdades em saúde, ${ }^{9}$ mesmo com os avanços obtidos com o SUS e com a expansão da Atenção Primária à Saúde (APS) pela Estratégia Saúde da Família (ESF), alguns desafios ainda demarcam a saúde pública brasileira, ${ }^{10}$ incluindo a centralização do atendimento médico, ${ }^{11}$ a concentração desses profissionais em grandes áreas urbanas ${ }^{12}$ de forma desigual e insuficiente, ${ }^{13,14}$ bem como as grandes desigualdades regionais que se refletem nas diferenças de atenção à saúde entre municípios brasileiros. ${ }^{15}$

Essas desigualdades surgiram a partir do processo histórico de construção da saúde pública e previdenciária ao longo do século $X X^{16}$ e culminaram na situação de extrema desigualdade entre entes federados quanto ao acesso a serviços médicos e à equidade de tais serviços. A partir da década de 1990, a implantação da ESF surge como estratégia horizontal de reorientar a APS no SUS, a qual mesmo tendo desenvolvido inúmeros avanços não conseguiu realizar a promoção da equidade vertical de modo tão expressivo. ${ }^{17}$ Dessa forma, alguns avanços ainda são prementes ao SUS, tais como: aumento da relação médico/população; investimentos na formação de médicos de família e comunidade; interiorização e fixação dos profissionais médicos, sobretudo em áreas de maior vulnerabilidade. ${ }^{12}$

Nesse sentido, o PMM representou uma estratégia de combate às desigualdades em saúde e buscou ampliar o acesso a esses serviços e sua equidade. ${ }^{12}$ Todavia, os efeitos desse programa não podem ser analisados apenas no contexto macropolítico, tendo em vista que, em cada estado, em cada município, o PMM assumiu características peculiares de implantação, que respondem ao questionamento de sua efetividade. ${ }^{18}$

Um dos estados brasileiros que recebeu profissionais do PMM foi o Espírito Santo (ES). Trata-se de um estado localizado na região Sudeste do Brasil e que possui área de $46.074,444 \mathrm{~km}^{2}$, população estimada para 2019 de 4.018.650 habitantes e 78 municípios. ${ }^{19}$ Caracteriza-se pela peculiaridade de apresentar, na capital Vitória, o menor percentual de extrema pobreza do estado $(1,54 \%)^{20}$ e a maior proporção, entre todas as capitais brasileiras, de médicos por habitantes — na razão de 12,27 médicos por mil habitantes, enquanto, nas cidades do interior do estado, essa razão não ultrapassa 1,5 médico por mil habitantes. ${ }^{13}$ Nessa linha, o Espírito Santo também apresenta desigualdades entre municípios, registrando populações que apresentam alto percentual de pobreza, como a de Brejetuba. ${ }^{20}$ Assim, este estudo teve como objetivo analisar as características da implantação do PMM nos 78 municípios do estado do Espírito Santo no primeiro ciclo do programa, de 2013 a 2016.

\section{MÉTODOS}

Trata-se de um estudo quantitativo, com base em dados secundários, no qual a implantação do PMM no Espírito Santo foi delineada com dados coletados da Secretaria de Estado da Saúde (SESA); do Cadastro Nacional de Estabelecimentos de Saúde (CNES), pertencentes ao Departamento de Informática do SUS (DATASUS); do Instituto Brasileiro de Geografia e Estatística (IBGE), para a coleta da população residente estimada; e do Instituto Jones dos Santos Neves (IJSN), para a verificação de população em extrema pobreza nos municípios do Espírito Santo. Os dados referem-se ao período de 2013 a 2016, correspondente ao primeiro ciclo de implantação do PMM.

A unidade de análise deste estudo inclui o agregado populacional e o espaço geográfico do município. Cada município corresponde a uma unidade elementar de análise. Um banco de dados foi construído considerando-se cada um dos 78 municípios, com suas respectivas variáveis. 
1. Porte populacional: reflete o agrupamento de municípios de acordo com o seu número de habitantes - municípios de até 10 mil habitantes; municípios de 10.001 a 20 mil habitantes; municípios de 20.001 a 50 mil habitantes; municípios de 50.001 a 200 mil habitantes; municípios de 200.001 habitantes ou mais.

2. Índice de Desenvolvimento Humano (IDH) municipal: corresponde ao índice de cada município para o ano de 2016. Trata-se de uma medida composta de três indicadores: a oportunidade de viver uma vida longa e saudável, de ter acesso ao conhecimento e de ter um padrão de vida que garanta as necessidades básicas, como saúde, educação e renda. Varia de 0 a 1, e quanto mais próximo de 1 maior o desenvolvimento humano. ${ }^{21}$

3. População em extrema pobreza: representa o percentual da população do município que vivia em extrema pobreza no ano de 2016, considerando-se como extrema pobreza a renda de US\$1,90 per capita por dia, estabelecida pelo Banco Mundial ao analisar países extremamente pobres. ${ }^{22}$

4. População usuária de plano privado de saúde: equivale à razão, expressa em porcentagem, entre o número de beneficiários de planos privados de saúde e a população residente,$^{23}$ para o ano de 2016.

5. População coberta pela ESF: representa a cobertura populacional estimada pelas equipes da ESF, dada pelo percentual da população coberta por essas equipes em relação à estimativa populacional, na qual se considera o parâmetro de três mil indivíduos cobertos por equipe, ${ }^{24}$ para o ano de 2016.

6. População coberta pela atenção primária: representa a cobertura populacional estimada na atenção primária, dada pelo percentual da população coberta por equipes da ESF e por equipes de atenção primária tradicional equivalentes e parametrizadas em relação à estimativa populacional ${ }^{24}$ para o ano de 2016.

7. População coberta pela saúde bucal: corresponde ao percentual da população do município coberta pela saúde bucal, no ano de 2016, a qual considera a soma da carga horária dos cirurgiões-dentistas do município dividida por 40 horas e multiplicada por três mil habitantes, sendo esse valor dividido pela estimativa populacional e multiplicado por uma base de $100 .{ }^{24}$

8. Total de médicos do PMM para os anos de 2013, 2014, 2015 e 2016: equivale ao número de médicos do PMM que cada município recebeu no ano de 2013, no ano de 2014, no ano de 2015 e no ano de 2016.

9. Total de médicos da ESF para os anos de 2012 e 2016: equivale ao número de médicos atuantes na ESF do município no ano de 2012 e no ano de 2016.

10. Adesão dos municípios ao PMM no período de 2013-2016: corresponde ao percentual de adesão dos municípios ao PMM em relação ao ano, que corresponde ao número de municípios aderidos dividido pelo total de municípios do estado.

Os dados foram analisados nos programas Microsoft Office Excel, versão 2010, e Statistical Package for the Social Sciences (SPSS), versão 21. Na análise descritiva, foram calculadas as frequências absolutas e relativas, além das médias e dos desvios padrão. Na análise inferencial, foram utilizados os testes de Kruskal-Wallis para realizar a comparação das médias e os testes de análise de variância (ANOVA) para verificar as diferenças das variâncias/desvios padrão entre as faixas populacionais.

Este estudo foi aprovado pelo Comitê de Ética em Pesquisa (CEP) com o número de Certificado de Apresentação para Apreciação Ética (CAAE) 58948516.5.0000.5060 e obteve autorização da SESA do Espírito Santo. 


\section{RESULTADOS}

No que diz respeito ao porte populacional, foram identificados nove municípios de até 10 mil habitantes; 30 municípios de 10.001 a 20 mil habitantes; 27 municípios de 20.001 a 50 mil habitantes; sete municípios de 50.001 a 200 mil habitantes; cinco municípios de 200.001 habitantes ou mais.

Em relação ao perfil dos municípios, no que se refere ao IDH, à população em extrema pobreza, à população usuária de plano privado de saúde, à população coberta pela Estratégia Saúde da Família, à população coberta pela atenção primária e à população coberta pela saúde bucal, evidenciam-se diferenças estatisticamente significantes para todos as variáveis avaliadas no perfil municipal entre os diferentes portes populacionais $(p<0,001)$. Isso mostra que as desigualdades municipais estão presentes diante da implantação do PMM, conforme a Tabela 1.

Tabela 1. Perfil sociodemográfico dos municípios que receberam médicos do Programa Mais Médicos. Espírito Santo, Brasil, 2013-2016.

\begin{tabular}{|c|c|c|c|c|c|c|c|}
\hline Porte populacional & Média & Desvio padrão (dp) & Percentil 25 & Mediana & Percentil 75 & p-valor* & p-valor ${ }^{\dagger}$ \\
\hline \multicolumn{8}{|c|}{ Índice de desenvolvimento humano municipal } \\
\hline Até 10.000 & 0,66 & 0,02 & 0,65 & 0,67 & 0,67 & & \\
\hline 10.001 a 20.000 & 0,69 & 0,03 & 0,66 & 0,69 & 0,71 & & \\
\hline 20.001 a 50.000 & 0,69 & 0,03 & 0,67 & 0,68 & 0,71 & $<0,001$ & 0,163 \\
\hline 50.001 a 200.000 & 0,73 & 0,02 & 0,71 & 0,73 & 0,75 & & \\
\hline 200.001 ou mais & 0,77 & 0,05 & 0,74 & 0,75 & 0,8 & & \\
\hline \multicolumn{8}{|c|}{ População em extrema pobreza (\%) } \\
\hline Até 10.000 & 9,05 & 3,69 & 7,79 & 9,42 & 9,76 & & \\
\hline 10.001 a 20.000 & 6,61 & 3,91 & 3,89 & 5,59 & 7,51 & & \\
\hline 20.001 a 50.000 & 6,14 & 1,79 & 4,95 & 5,96 & 7,01 & $<0,001$ & 0,182 \\
\hline 50.001 a 200.000 & 4,03 & 1,33 & 3,38 & 3,91 & 5,19 & & \\
\hline 200.001 ou mais & 2,35 & 0,77 & 1,62 & 2,4 & 2,95 & & \\
\hline \multicolumn{8}{|c|}{ População usuária de plano privado de saúde (\%) } \\
\hline Até 10.000 & 6,94 & 4,71 & 3,77 & 6,23 & 7,76 & & \\
\hline 10.001 a 20.000 & 12,14 & 7,17 & 7,78 & 11,58 & 13,98 & & \\
\hline 20.001 a 50.000 & 12,89 & 4,98 & 8,48 & 12,34 & 17,16 & $<0,001$ & 0,073 \\
\hline 50.001 a 200.000 & 28,1 & 7,15 & 23,66 & 26,65 & 36,95 & & \\
\hline 200.001 ou mais & 44,41 & 14,85 & 33,65 & 39,67 & 50,17 & & \\
\hline \multicolumn{8}{|c|}{ População coberta pela Estratégia Saúde da Família (\%) } \\
\hline Até 10.000 & 97,68 & 6,97 & 100 & 100 & 100 & & \\
\hline 10.001 a 20.000 & 97,11 & 9,94 & 100 & 100 & 100 & & \\
\hline 20.001 a 50.000 & 88,27 & 16,72 & 76 & 100 & 100 & $<0,001$ & $<0,001$ \\
\hline 50.001 a 200.000 & 73,88 & 18,99 & 60,79 & 66,79 & 100 & & \\
\hline 200.001 ou mais & 45,41 & 32,68 & 20,3 & 33,47 & 79,74 & & \\
\hline \multicolumn{8}{|c|}{ População coberta pela Atenção Primária à Saúde (\%) } \\
\hline Até 10.000 & 100 & 0 & 100 & 100 & 100 & & \\
\hline 10.001 a 20.000 & 99,73 & 1,46 & 100 & 100 & 100 & & \\
\hline 20.001 a 50.000 & 95,52 & 8,04 & 93 & 100 & 100 & $<0,001$ & 0,001 \\
\hline 50.001 a 200.000 & 83,14 & 20,55 & 57 & 95 & 100 & & \\
\hline 200.001 ou mais & 75,8 & 23,3 & 65 & 73 & 97 & & \\
\hline
\end{tabular}


Tabela 1. Continuação.

\begin{tabular}{|c|c|c|c|c|c|c|c|}
\hline Porte populacional & Média & Desvio padrão (dp) & Percentil 25 & Mediana & Percentil 75 & p-valor* & p-valor ${ }^{\dagger}$ \\
\hline \multicolumn{8}{|c|}{ População coberta pela saúde bucal (\%) } \\
\hline Até 10.000 & 82,11 & 31,94 & 84,65 & 100 & 100 & & \\
\hline 10.001 a 20.000 & 90,33 & 25,71 & 96,38 & 100 & 100 & & \\
\hline 20.001 a 50.000 & 78,43 & 27,13 & 59,97 & 85,07 & 100 & $<0,001$ & $<0,001$ \\
\hline 50.001 a 200.000 & 61,27 & 12,68 & 57,73 & 62,93 & 72,1 & & \\
\hline 200.001 ou mais & 50,25 & 17,73 & 37,45 & 51,12 & 59,72 & & \\
\hline
\end{tabular}

${ }^{*}$ Teste de Kruskal-Wallis; †Teste ANOVA.

A análise do IDH revela que a média foi maior para os municípios com 200.001 habitantes ou mais, diferentemente dos municípios com até 10 mil habitantes (média=0,66 $\pm 0,02 \mathrm{dp}$ ) e dos municípios com 50.001

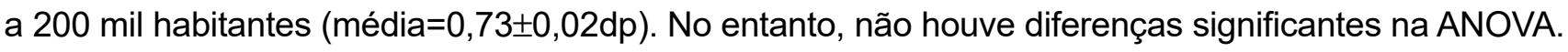

No que tange à variável percentual de extrema pobreza, foi possível observar que os municípios com menor porte populacional apresentaram maior taxa de pobreza, haja vista a média igual a 6,61 $\pm 3,91 \mathrm{dp}$ para

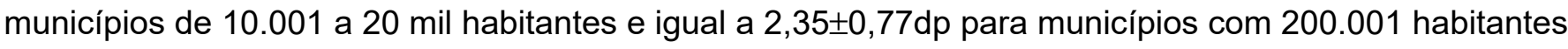
ou mais, sem significância na testagem de variância.

Em consonância com os dados anteriores, municípios maiores apresentaram taxa de população usuária de plano privado de saúde também maior, destacando-se aqueles com 200.001 habitantes ou mais, que atingiram a média de $44,41 \pm 14,85 \mathrm{dp}$, assim como apresentaram a maior média entre os municípios que fazem parte desse grupo. Esse dado se contrapôs ao dos municípios com até 10 mil habitantes, que exibiram média de 6,94 $\pm 4,71 \mathrm{dp}$, e ao dos municípios com 20.001 a 50 mil habitantes, que apresentaram média igual a 12,89 $\pm 4,98 \mathrm{dp}$.

Em relação à cobertura de ESF, os resultados mostram que municípios de menor porte populacional, com até 10 mil habitantes, apresentaram ampla cobertura do programa (média=97,68 $\pm 6,97 \mathrm{dp}$ ), enquanto municípios com 200.001 habitantes ou mais apresentaram cobertura muito inferior (média=45,41 $\pm 32,68 \mathrm{dp}$ ). Além disso, a variabilidade para as duas faixas populacionais reitera a grande disparidade de cobertura da ESF entre os municípios de um mesmo grupo populacional, disparidade que se mostra progressiva com o aumento da faixa populacional.

Na sequência, a cobertura da atenção primária é total entre os municípios de até 10 mil habitantes, configurando uma abrangência ampla e homogênea nessa faixa populacional, enquanto os municípios

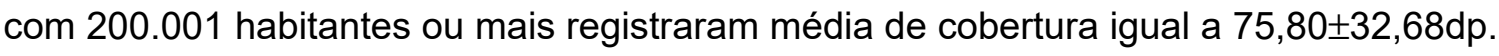

No que se refere à cobertura da população em relação à saúde bucal, o melhor desempenho foi registrado para os municípios com população de 10.001 a 20 mil (média=90,33 $\pm 25,71 \mathrm{dp}$ ) e para os municípios com até 10 mil habitantes (média=82,11 $\pm 31,94 \mathrm{dp}$ ).

A Tabela 2 mostra que a comparação das médias também revelou valores significativos quanto ao número de médicos do PMM de 2013 a 2016. Esses dados mostram que houve aumento progressivo no número de médicos desde a implantação do programa, em 2013, até 2016, com aumento na média para todas as faixas populacionais.

Entretanto, os dados também evidenciaram que, mesmo com o aumento do número de médicos, a diferença de médias entre municípios da mesma faixa populacional também foi maior. Verificou-se que, no primeiro ano de implantação do PMM, 2013, os municípios de porte populacional com até 10 mil habitantes 
Tabela 2. Evolução da inserção de médicos nos municípios. Espírito Santo, Brasil, 2013-2016.

\begin{tabular}{|c|c|c|c|c|c|c|c|}
\hline Porte populacional & Média & Desvio padrão & Percentil 25 & Mediana & Percentil 75 & p-valor* & p-valor ${ }^{\dagger}$ \\
\hline \multicolumn{8}{|c|}{ Total de médicos no Programa Mais Médicos-2013 } \\
\hline Até 10.000 & 0,11 & 0,33 & 0 & 0 & 0 & & \\
\hline 10.001 a 20.000 & 0,1 & 0,4 & 0 & 0 & 0 & & \\
\hline 20.001 a 50.000 & 0,19 & 0,4 & 0 & 0 & 0 & $<0,001$ & $<0,001$ \\
\hline 50.001 a 200.000 & 2,14 & 3,76 & 0 & 0 & 4 & & \\
\hline 200.001 ou mais & 13,2 & 11,69 & 5 & 13 & 18 & & \\
\hline \multicolumn{8}{|c|}{ Total de médicos no Programa Mais Médicos-2014 } \\
\hline Até 10.000 & 0,33 & 0,5 & 0 & 0 & 1 & & \\
\hline 10.001 a 20.000 & 1,1 & 1,37 & 0 & 1 & 2 & & \\
\hline 20.001 a 50.000 & 3,07 & 2,51 & 1 & 3 & 5 & $<0,001$ & $<0,001$ \\
\hline 50.001 a 200.000 & 13,57 & 5,13 & 11 & 14 & 17 & & \\
\hline 200.001 ou mais & 34,6 & 26,85 & 23 & 24 & 28 & & \\
\hline \multicolumn{8}{|c|}{ Total de médicos no Programa Mais Médicos-2015 } \\
\hline Até 10.000 & 0,33 & 0,5 & 0 & 0 & 1 & & \\
\hline 10.001 a 20.000 & 1,27 & 1,34 & 0 & 1 & 2 & & \\
\hline 20.001 a 50.000 & 3,48 & 2,46 & 1 & 3 & 6 & $<0,001$ & $<0,001$ \\
\hline 50.001 a 200.000 & 15,57 & 8,4 & 10 & 14 & 22 & & \\
\hline 200.001 ou mais & 37 & 22,28 & 23 & 29 & 34 & & \\
\hline \multicolumn{8}{|c|}{ Total de médicos no Programa Mais Médicos-2016 } \\
\hline Até 10.000 & 0,33 & 0,5 & 0 & 0 & 1 & & \\
\hline 10.001 a 20.000 & 1,23 & 1,22 & 0 & 1 & 2 & & \\
\hline 20.001 a 50.000 & 3,48 & 2,59 & 1 & 3 & 6 & $<0,001$ & $<0,001$ \\
\hline 50.001 a 200.000 & 17,43 & 6,11 & 14 & 16 & 23 & & \\
\hline 200.001 ou mais & 35,2 & 23,97 & 24 & 24 & 33 & & \\
\hline \multicolumn{8}{|c|}{ Total de médicos na Estratégia Saúde da Família-2012 } \\
\hline Até 10.000 & 1,11 & 0,93 & 0 & 1 & 2 & & \\
\hline 10.001 a 20.000 & 1,37 & 1,07 & 1 & 1 & 2 & & \\
\hline 20.001 a 50.000 & 3,22 & 2,21 & 1 & 3 & 4 & $<0,001$ & $<0,001$ \\
\hline 50.001 a 200.000 & 7,43 & 6,24 & 1 & 5 & 12 & & \\
\hline 200.001 ou mais & 22,8 & 19,12 & 11 & 18 & 26 & & \\
\hline \multicolumn{8}{|c|}{ Total de médicos na Estratégia Saúde da Família-2016 } \\
\hline Até 10.000 & 1,78 & 0,67 & 1 & 2 & 2 & & \\
\hline 10.001 a 20.000 & 3,1 & 1,37 & 2 & 3 & 4 & & \\
\hline 20.001 a 50.000 & 5,11 & 3 & 2 & 5 & 8 & $<0,001$ & $<0,001$ \\
\hline 50.001 a 200.000 & 19,57 & 7,66 & 12 & 22 & 25 & & \\
\hline 200.001 ou mais & 33,6 & 9,66 & 32 & 32 & 35 & & \\
\hline
\end{tabular}

${ }^{*}$ Teste de Kruskal-Wallis; 'Teste ANOVA.

apresentaram menor total de médicos do PMM (média $=0,11 \pm 0,33 \mathrm{dp}$ ), com aumento (média $=0,33 \pm 0,5 \mathrm{dp}$ ) no ano de 2014 e ausência de alteração até 2016. Os municípios com 200.001 habitantes ou mais apresentaram média igual a 13,20 $\pm 11,69 \mathrm{dp}$ no primeiro ano, que quase triplicou no ano de 2014 (média=34,60 $\pm 26,85 \mathrm{dp}$ ), sofreu acréscimo no ano de 2015 (média=37,00 $\pm 22,28 \mathrm{dp}$ ) e teve queda em 2016 (média=35,20 $\pm 23,97 \mathrm{dp}$ ). 
A Tabela 2 também apresenta as médias do número de médicos na ESF em 2012 (antes de o PMM ser implantado) e em 2016 (após a implantação do PMM) em relação ao porte populacional. Verifica-se aumento na média de médicos do programa do ano de 2012 para o ano de 2016, em todas as faixas populacionais. Além disso, municípios com até 10 mil habitantes exibiram redução na variabilidade interna

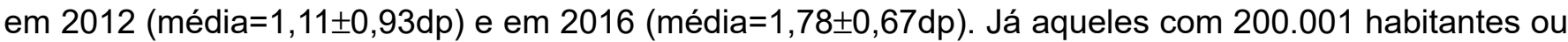
mais apresentaram grande diminuição na variabilidade entre seus municípios após a implantação do PMM, uma vez que a variabilidade teve queda considerável de 2012 (média=22,80 1 19,12dp) para 2016 (média=33,60 $\pm 9,66 \mathrm{dp}$ ).

Quanto à adesão dos municípios ao PMM, o Gráfico 1 demonstra como isso ocorreu, por porte populacional, durante o período de 2013 a 2016. Observou-se que houve maior adesão, no início, entre os municípios de maior porte populacional, com adesão aproximada de 85 e 80\% (50.001 a 200 mil e 200.001 habitantes ou mais, respectivamente). Essa adesão atingiu o percentual de adesão de $100 \%$ no ano de 2014 e manteve-se nele nos anos de 2015 e 2016.

Já os municípios com até 10 mil habitantes tiveram alta adesão em 2013 (aproximadamente 70\%), sofreram grande queda da adesão ao PMM em 2014 e mantiveram o percentual em torno de $30 \%$ nos

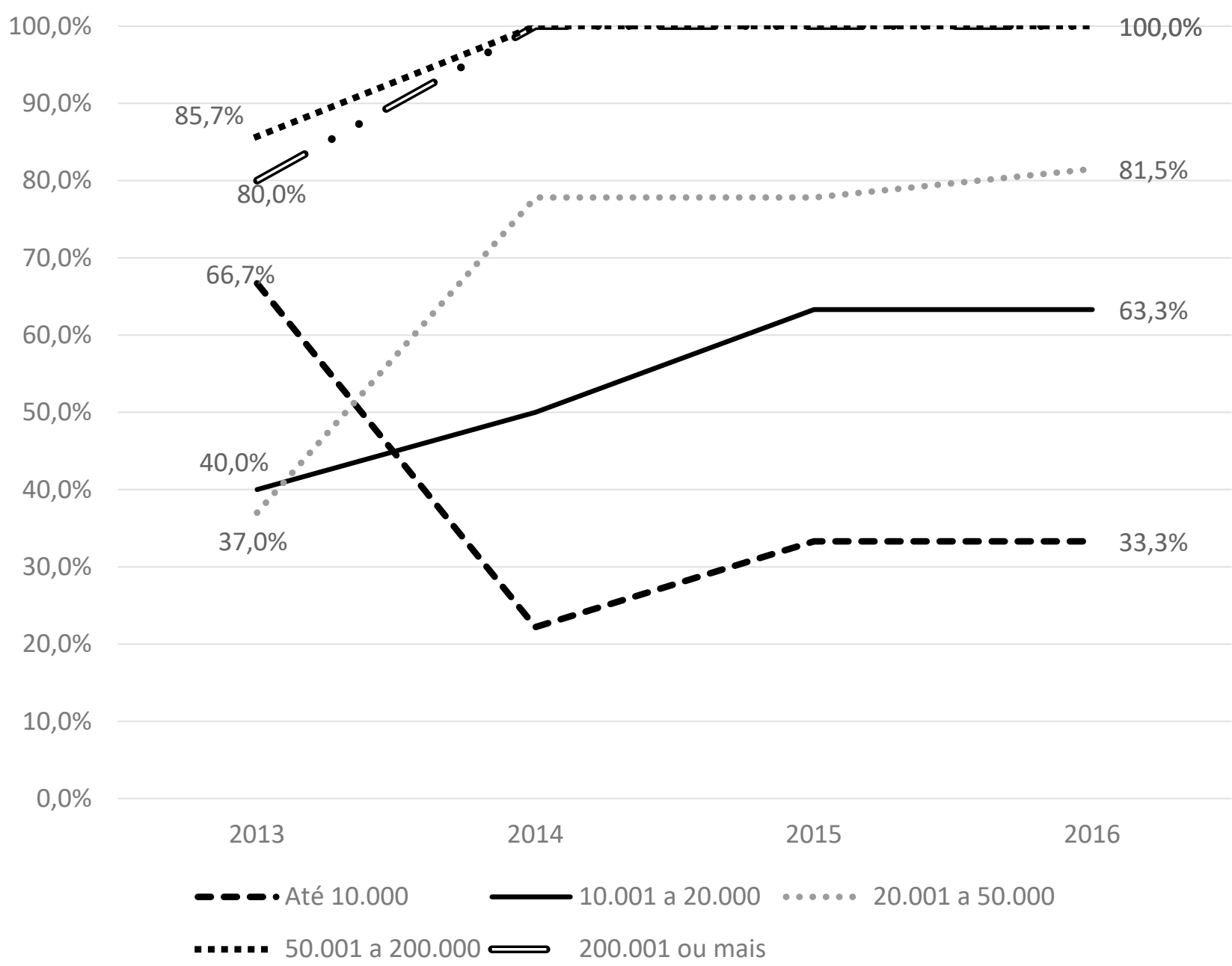

Gráfico 1. Adesão dos municípios ao Programa Mais Médicos. Espírito Santo, Brasil, 2013-2016. 
anos de 2015 e 2016. Em oposição, 40\% dos municípios com 10.001 a 20 mil habitantes aderiram ao programa em 2013, aumentaram para aproximadamente 60\% em 2014 e mantiveram-se nessa média até 2016. Os municípios com 20.001 a 50 mil habitantes também iniciaram em 2013 com adesão aproximada de $40 \%$, mas exibiram aumento considerável em 2014 (chegando a quase $80 \%$ ) e encerraram o primeiro ciclo do programa com permanência da alta adesão.

\section{DISCUSSÃO}

Por meio do presente estudo, evidenciaram-se as desigualdades existentes em um estado brasileiro de pequena área territorial, de acordo com o porte populacional municipal, quanto ao IDH, à população em extrema pobreza e à cobertura de serviços de saúde durante a implantação do PMM. Assim, a análise da implantação do PMM no Espírito Santo traz a reflexão de que, mesmo em um estado de pequenas dimensões geográficas e baixo contingente populacional, desigualdades fazem-se presentes. Essa reflexão abarca a discussão do modelo de federalismo adotado no Brasil e demarca as características estruturais dessa desigualdade, que trouxe reflexo em todas as áreas, ${ }^{25}$ assim como na gestão da saúde no SUS, principalmente pela descentralização dos recursos fiscais da União e da intensa emancipação política. ${ }^{6}$ Essas profundas desigualdades, além de desafiarem o SUS, comprometem o desempenho do sistema de saúde. ${ }^{26}$

Com a Constituição de 1988, o Brasil tornou-se uma República Federativa com regime democrático, que buscou a descentralização e a autonomia dos entes federados. Entretanto, ainda permaneceram indefinidas as competências específicas da União, dos estados e municípios, o que tornou essa relação complexa, ${ }^{27}$ sem uma coordenação estratégica. ${ }^{6}$ Nesse sentido, o financiamento brasileiro na área da saúde foi demarcado pela centralização da União, pela escassez e pela vulnerabilidade ao sistema econômico, além de pela presença de desigualdades em sua destinação. ${ }^{28}$ Ao mesmo tempo que os municípios foram fortalecidos com maior autonomia e proximidade com a população, ${ }^{27}$ a variação econômica regional e a disparidade entre encargos e receitas fizeram com que alguns municípios não conseguissem assumir sozinhos tamanha responsabilidade de gestão. ${ }^{6}$

Na saúde, se, por um lado, pequenos municípios possuem facilidade na participação social, ${ }^{29}$ por outro, quando não dispõem de programas de saúde do governo que possibilitem o aumento dos fundos, ${ }^{5}$ a restrição financeira dificulta a gestão, a oferta de recursos para a seleção e a fixação de profissionais, impossibilitando desde o desenvolvimento ${ }^{29}$ até a ampliação do acesso à atenção primária e à ESF. ${ }^{30}$

Nesse aspecto, as desigualdades também podem se manifestar em um mesmo município, exemplificando a existência de dois mundos; isso porque, enquanto existem as melhores estruturas na área central de um município, ${ }^{31} \mathrm{com}$ a concentração de profissionais médicos nessas áreas urbanas ${ }^{12} \mathrm{e}$ requisitos que garantem a qualidade de vida, do outro se faz presente uma periferia formada de famílias de baixa renda, com carências estruturais básicas ${ }^{31}$ e dificuldade de fixação de médicos nessas áreas de vulnerabilidade. ${ }^{12}$

Reforçando esse entendimento, a descentralização do poder e a transferência da responsabilidade dos serviços de saúde para os municípios, sem padronização e equalização de como aconteceria essa oferta, ${ }^{7}$ aliadas à extrema heterogeneidade socioeconômica do país, contribuíram potencialmente para diferenças inter-regionais e intrarregionais, ${ }^{6}$ além de demarcarem a desigualdade intermunicipal. ${ }^{7}$ Essas desigualdades são evidentes no cenário de implantação do PMM no Espírito Santo, seja pelo maior percentual de população em extrema pobreza em municípios de menor porte populacional, seja 
pelas disparidades de cobertura da ESF entre municípios de mesmo porte e entre municípios de portes populacionais distintos.

Assim como ocorre em outros estados da federação brasileira, no estado do Espírito Santo existem discrepâncias quanto ao crescimento econômico que corroboram seu desenvolvimento desigual. ${ }^{32}$ Exemplificando essa realidade, o município de Brejetuba destaca-se como o de maior percentual de população em extrema pobreza no ano de 2016: trata-se de um município do interior do estado, que apresenta população estimada de 12.797 habitantes, ${ }^{33}$ IDH em 2016 de 0,66, e que conta com cobertura de $100 \%$ de ESF. Em contrapartida, Vitória representa o município com menor percentual de população em extrema pobreza no mesmo período: apresenta população estimada de 359.555 habitantes, ${ }^{34}$ IDH em 2016 de 0,85 e $79,74 \%$ de cobertura de ESF.

Nesse contexto, a análise do presente estudo demonstrou que os municípios com menor porte populacional apresentaram maior percentual de pobreza, destacando-se as populações que vivem em regiões mais distantes das capitais e de difícil acessibilidade geográfica. ${ }^{35}$ Esse achado confirma que, muitas vezes, apenas apresentar o serviço não significa garantir a possibilidade de acesso a ele, ${ }^{17}$ o que corrobora a persistente dificuldade de fixação de médicos em áreas mais vulneráveis e de difícil acesso do país. ${ }^{14}$

No cenário brasileiro, sabe-se que o cadastramento em unidades de saúde da família é maior na área rural $(70,9 \%)$ do que na área urbana $(50,6 \%)$, o que reitera a necessidade de cobertura de saúde às populações mais vulneráveis. ${ }^{36}$ Entretanto, no presente estudo, a adesão ao PMM foi grande nos municípios menores apenas no início do programa, tornando-se estável em seguida e permanecendo baixa nos anos de 2015 e 2016, enquanto, nos municípios de maior porte populacional, houve contínuo crescimento da adesão, evidenciando a dificuldade de permanência de municípios de pequeno porte populacional no PMM. Essa dificuldade pode ser proveniente dos obstáculos de manutenção financeira das contrapartidas municipais. Entre as várias atribuições dos municípios, uma era a de custear a moradia e a alimentação dos médicos participantes, aliada à de disponibilizar deslocamento e água potável aos profissionais $^{37}$ - assim como mencionado no estado do Maranhão, no qual se ressaltou a dificuldade de gestão local de alguns municípios por apresentarem limitações na garantia dessas contrapartidas. ${ }^{38}$

Reforçando esse cenário encontrado na implantação do PMM no Espírito Santo, municípios que apresentaram a possibilidade de sustentar os benefícios trazidos com o PMM foram capazes de potencializá-lo, enquanto municípios menores, que apresentaram limitação financeira, sofreram em intensidade maior os efeitos da política de saúde central e hierárquica. ${ }^{39} \mathrm{Com}$ isso, reafirmou-se o quanto o contexto geopolítico é capaz de interferir no índice de pobreza de um município ${ }^{40}$ e como as barreiras geográficas e financeiras podem contribuir para a obstrução do consumo dos serviços. ${ }^{17}$

Em contrapartida às limitações do programa nos municípios menores, observou-se, neste estudo, que o PMM reduziu as diferenças no número de médicos entre os municípios de faixas populacionais maiores, como nos de 200.001 habitantes ou mais, potencializando o acesso ao sistema de saúde. Isso possibilitou a inserção de milhões de pessoas no SUS, a regularidade dos profissionais médicos que cumprem carga horária determinada e o compromisso com o vínculo de trabalho desses profissionais, ${ }^{41}$ proporcionando, assim, a fixação de médicos em áreas de maior vulnerabilidade social. ${ }^{11}$

Nesse aspecto, a intervenção do Ministério da Saúde (MS), principalmente por meio do incentivo financeiro, ${ }^{42}$ demonstrou influência na adesão dos municípios ao $\mathrm{PMM}^{43}$ e possibilitou a ampliação da presença de profissionais médicos no programa, aumentando e completando equipes incompletas ${ }^{41}$ e proporcionando à população assistida pelo SUS o acesso aos serviços de saúde. ${ }^{39}$ Isso é demonstrado 
em nosso estudo pela ampla cobertura da APS e pelo aumento do número de profissionais médicos participantes do PMM.

No entanto, apesar dos avanços observados com o PMM, ainda permanecem importantes diferenças na cobertura populacional da ESF, ${ }^{41}$ o que foi reforçado no presente estudo pela baixa cobertura da ESF nos municípios de 200.001 habitantes ou mais do Espírito Santo. Nesse sentido, a variação nos processos organizacionais resulta em diferentes implantações locais, interferindo diretamente na cobertura, no acesso e nos resultados alcançados, de forma que a fragmentação e a descentralização dos serviços do SUS continuam desafiando as ações de integração das redes dos serviços de saúde. ${ }^{44}$ Isso pôde ser observado pela discrepância apresentada nesse grupo de mesmo porte populacional, que demarca as diversidades encontradas nos serviços de saúde da APS. ${ }^{29}$

Em vista disso, apresentar cobertura de APS não significa garantir a prestação de atenção e cuidado, pois, além da estrutura, algumas dimensões - como processo de trabalho, fluxos, acessibilidade, ${ }^{45}$ organização da unidade básica para a oferta de serviços com equidade e integralidade — devem se fazer presentes ${ }^{29}$ reafirmando que a possibilidade de oferta ou bloqueio desses serviços está diretamente relacionada a barreiras organizacionais, informacionais e culturais. ${ }^{17}$

Nesse sentido, o presente estudo reitera que o desafio da conquista da equidade pela ESF, somado à fragmentação do sistema de financiamento e à diminuição da participação dos recursos federais no custeio da saúde pública, atrelada ao aumento do papel exercido pelos municípios na política de saúde ${ }^{46}$ tem demonstrado a dificuldade de os municípios fazerem a própria gestão. Isso, tendo em vista a formulação e a implantação linear das políticas, que não leva em consideração a heterogeneidade e as dificuldades específicas de cada local, ${ }^{39}$ assim como pôde ser percebido com a variabilidade encontrada na implantação do PMM no estado do Espírito Santo.

Ao considerarmos a heterogeneidade existente no acesso desigual ao SUS, de acordo com a menor possibilidade de acesso aos serviços de saúde em relação ao porte populacional, faz-se necessária uma abordagem sobre a equidade. ${ }^{47}$ Dessa forma, para a obtenção de êxito na diminuição das desigualdades em saúde, é preciso políticas públicas justas, ${ }^{48}$ de forma a existirem a cooperação e a interação da variedade encontrada nos diversos grupos sociais existentes. ${ }^{49} \mathrm{Com}$ isso, objetiva-se a diminuição das desigualdades de acesso, garantindo que as pessoas com menor poder aquisitivo, que muitas vezes apresentam maior necessidade do consumo de serviços de saúde, consigam acessá-los. ${ }^{10}$

Destaca-se que no Espírito Santo, de 2013 a 2016, a média do número de médicos que participaram do PMM aumentou de acordo com o porte populacional e foi muito maior nos municípios maiores, contrapondo alguns critérios estabelecidos para a alocação dos médicos do PMM. Segundo tais critérios, deveriam ser priorizadas áreas de difícil acesso, de difícil suprimento de médicos ou que possuíssem populações em situação de maior vulnerabilidade. ${ }^{50}$ Entretanto, também se deve presumir que, nos municípios maiores, haja populações que vivem em favelas e situações precárias, de vulnerabilidade, além de maior capacidade de absorver mais profissionais. Assim, mesmo estando em regiões próximas ao meio urbano e com alta densidade demográfica, havia dificuldades de fixar médicos nas periferias por estas caracterizarem áreas com maior índice de violência, como na realidade vivenciada pelo estado do Rio de Janeiro. ${ }^{51}$

A partir de 2018, um novo cenário político no país levou a reformulações no provimento de profissionais médicos, o que culminou com a substituição do PMM pelo Programa "Médicos pelo Brasil" em dezembro de 2019. O objetivo era possibilitar um vínculo trabalhista mais atrativo e estável com a assinatura da carteira de trabalho, além de satisfazer alguns interesses conflituosos que o PMM havia gerado. ${ }^{52}$ 
No que tange às limitações deste estudo, cabe ressaltar que, por ele trabalhar com dados secundários, o que possibilita maior abrangência populacional e baixo custo, ele também apresenta fragilidades pela falta de controle sobre os dados coletados e pela clareza desses dados quanto à cobertura da ESF para garantir a presença do profissional médico ou não, restringindo-se a um cenário de implantação no estado do Espírito Santo. Entretanto, o trabalho demonstra potencial ao contribuir como exemplo de análise do PMM em outros estados brasileiros, apresentando peculiaridades locais e regionais que podem contribuir para a política pública de saúde do Brasil.

\section{CONCLUSÕES}

Os resultados deste estudo demonstraram que a implantação do PMM no Espírito Santo contribuiu para o fortalecimento da APS, proporcionando o aumento do provimento dos profissionais médicos em municípios tanto de pequeno quanto de maior porte populacional e possibilitando o acesso da população a esses profissionais. Assim, mesmo que algumas desigualdades se tenham mantido de acordo com o porte populacional, o programa cumpriu a sua missão em favorecer a fixação médica.

Nesse aspecto, observou-se que o PMM no Espírito Santo teve maior capacidade de reduzir as desigualdades de saúde, mas não foi capaz de reduzir as desigualdades verticais, as quais estão diretamente relacionadas ao financiamento. Ressalta-se a necessidade de organização da gestão da APS no SUS de forma a contemplar a localização e as características regionais e socioeconômicas dos municípios, mediante a insolvência econômica e a incapacidade de investimento de alguns. Isso sugere a necessidade de um novo modelo de financiamento, além de maior participação do Estado no esforço pela diminuição das desigualdades no acesso à saúde e na integração dos serviços, bem como na consolidação do compartilhamento dos entes federativos na busca por um SUS resolutivo e equânime, independentemente do porte populacional.

\section{CONFLITO DE INTERESSES}

Nada a declarar.

\section{CONTRIBUIÇÕES DOS AUTORES}

Moraes, P.L.: Curadoria de dados, Escrita - primeira redação, Investigação, Recursos, Software, Visualização. Emerich, T.B.: Curadoria de dados, Escrita - primeira redação, Metodologia, Validação. Oliveira, A.E.: Administração do projeto, Conceituação, Supervisão. Santos Neto E.T.: Análise formal, Escrita - revisão e edição, Software, Supervisão.

\section{REFERÊNCIAS}

1. Santos W, Comes Y, Pereira LL, Costa AM, Merchan-Hamann E, Santos LMP. Avaliação do Programa Mais Médicos: relato de experiência. Saúde Debate 2019;43(120):256-68. https://doi.org/10.1590/0103-1104201912019

2. Parahos R, Figueiredo Filho DB, Rocha EC, Silva Jr JA, Maia RG. Construindo indicadores sociais: uma revisão da bibliografia especializada. Perspectivas: Revista de Ciências Sociais 2013;44:147-73.

3. Barreto ML. Desigualdades em saúde: uma perspectiva global. Ciência \& Saúde Coletiva 2017;22(7):2097-108. https://doi. org/10.1590/1413-81232017227.02742017

4. Barata RB. Como e por que as desigualdades sociais fazem mal à saúde. Rio de Janeiro: Fiocruz; 2009. 
5. Rodrigues Filho J. A distribuição dos recursos de saúde no Brasil: a administração da desigualdade. Rev Adm Empr 1987;27(3):52-7. https://doi.org/10.1590/S0034-75901987000300007

6. Affonso RBA. Descentralização e reforma do Estado: a Federação brasileira na encruzilhada. Economia e Sociedade 2000;9(1):127-52.

7. Marques E, Arretche M. Condicionantes locais da descentralização das políticas de saúde. Caderno CRH 2003;39:55-81. https://doi.org/10.9771/ccrh.v16i39.18637

8. Travassos C. Eqüidade e o Sistema Único de Saúde: uma contribuição para debate. Cad Saúde Pública 1997;13(2):325-30. https://doi.org/10.1590/S0102-311X1997000200024

9. Norman AH. Estratégias que viabilizam o acesso aos serviços de Atenção Primária à Saúde no Reino Unido. Revista Brasileira de Medicina de Família e Comunidade 2019;14(41):1945. https://doi.org/10.5712/rbmfc14(41)1945

10. Neri M, Soares W. Desigualdade social e saúde no Brasil. Cad Saúde Pública 2002;18(Suplemento):77-87. https://doi. org/10.1590/S0102-311X2002000700009

11. Conill EM. Ensaio histórico-conceitual sobre a Atenção Primária à Saúde: desafios para a organização de serviços básicos e da Estratégia Saúde da Família em centros urbanos no Brasil. Cad Saúde Pública 2008;24(Sup 1):S7-S27. https://doi. org/10.1590/S0102-311X2008001300002

12. Tesser CD, Norman AH, Vidal TB. Acesso ao cuidado na Atenção Primária à Saúde brasileira: situação, problemas e estratégias de superação. Saúde Debate 2018;42(especial 1):361-78. https://doi.org/10.1590/0103-11042018S125

13. Scheffer M, Cassenote A, Guilloux AGA, Biancarelli A, Miotto BA, Mainardi GM. Demografia médica no Brasil 2018. São Paulo: Departamento de Medicina Preventiva da Faculdade de Medicina da USP; Conselho Regional de Medicina do Estado de São Paulo; Conselho Federal de Medicina; 2018. Disponível em: https://jornal.usp.br/wp-content/uploads/ DemografiaMedica2018.pdf

14. Pinto HA, Oliveira FP, Santana JSS, Santos FOS, Araujo SQ, Figueiredo AM, et al. Programa Mais Médicos: avaliando a implantação do Eixo Provimento de 2013 a 2015. Interface 2017;21(Supl 1):1087-101. https://doi.org/10.1590/180757622016.0520

15. Viacava F, Oliveira RAD, Carvalho CC, Laguardia J, Bellindo JG. SUS: oferta, acesso e utilização de serviços de saúde nos últimos 30 anos. Ciênc Saúde Colet 2018;23(6):1751-62. https://doi.org/10.1590/1413-81232018236.06022018

16. Carvalho G. A saúde pública no Brasil. Estud Av 2013;27(78):7-26. https://doi.org/10.1590/S0103-40142013000200002

17. Travassos C, Castro MSM. Determinantes e desigualdades sociais no acesso e na utilização de serviços de saúde. In: Giovanella L, Escorel S, Lobato LVC, Noronha JC, Carvalho Al. Políticas e sistemas de saúde no Brasil. Rio de Janeiro: Fiocruz; 2014. p. 183-206.

18. Organização Pan-Americana da Saúde. Implementação do Programa "Mais Médicos" em Curitiba. Experiências inovadoras e lições aprendidas. Brasília: Organização Pan-Americana da Saúde; 2015. Disponível em: https://iris.paho.org/bitstream/ handle/10665.2/28568/9788579671050_por.pdf?sequence=1\&isAllowed=y

19. Instituto Brasileiro de Geografia e Estatística. Cidades e Estados. Espírito Santo [Internet]. 2019 [acessado em 13 abr. 2020$].$ Disponível em: https://www.ibge.gov.br/cidades-e-estados/es/.html.

20. Governo do Espírito Santo. Espírito Santo em dados [Internet]. [acessado em 25 mar. 2020]. Disponível em: https://www. es.gov.br/es-em-dados.

21. Programa das Nações Unidas para o Desenvolvimento. Índice de Desenvolvimento Humano. O que é o IDHM? [Internet] [acessado em 27 abr. 2020]. Disponível em: https://www.br.undp.org/content/brazil/pt/home/idh0/conceitos/o-que-e-o-idhm.html

22. Governo do Espírito Santo. Instituto Jones dos Santos Neves. Perfil da pobreza no Espírito Santo: famílias inscritas no CadÚnico 2019. [acessado em 15 out. 2021] Disponível em: http://www.ijsn.es.gov.br/artigos/5437-perfil-da-pobreza-noespirito-santo-familias-inscritas-no-cadunico-2019

23. Agência Nacional de Saúde Suplementar. Informações em saúde suplementar. Taxa de cobertura [Internet]. 2020 [acessado em 28 abr. 2020]. Disponível em: http://www.ans.gov.br/anstabnet/notas_taxa_cobertura.htm

24. Brasil. Ministério da Saúde. Secretaria de Gestão Estratégica e Participativa. Departamento de Articulação Interfederativa. Caderno de Diretrizes, Objetivos, Metas e Indicadores: 2013-2015. Brasília: Ministério da Saúde; 2014 [acessado em 27 abr. 2020]. Disponível em: https://bvsms.saude.gov.br/bvs/publicacoes/caderno_diretrizes_objetivos_2013_2015_2edicao.pdf

25. Zechim P, Holanda FRB. Atributos espaciais da desigualdade nas grandes cidades brasileiras: uma relação entre segregação e morfologia. Cad Metropole 2019;21(44):55-78. https://doi.org/10.1590/2236-9996.2019-4403

26. Avaliação de Desempenho do Sistema de Saúde. Indicadores para o monitoramento do setor saúde na agenda 2030 para o desenvolvimento sustentável [Internet]. 2018 [acessado em 15 jul. 2019]. Disponível em: https://www.proadess.icict.fiocruz. br/Boletim_3_PROADESS_Agenda\%202030_agosto2018.pdf.

27. Goya HM. O pacto federativo brasileiro e os impactos desse modelo na gestão dos entes federados [monografia]. São Paulo: Insper Instituto de Estudo e Pesquisa da Faculdade de Economia e Administração; 2016. [acessado em 27 mar. 2020]. Disponível em: http://dspace.insper.edu.br/xmlui/bitstream/handle/11224/1563/Henrique\%20Molfi\%20Goya_Trabalho. pdf?sequence=1

28. Porto SM. Equidade na distribuição geográfica de recursos em saúde: uma contribuição para o caso brasileiro [tese]. Rio de Janeiro: Escola Nacional de Saúde Pública Sergio Arouca, Fundação Oswaldo Cruz; 1997 [acessado em 25 abr 2020]. Disponível em: https://www.arca.fiocruz.br/bitstream/icict/4522/2/147.pdf

29. Campos GWS, Pereira Júnior N. A atenção primária e o Programa Mais Médicos do Sistema Único de Saúde: conquistas e limites. Ciênc Saúde Colet 2016;21(9):2655-663. https://doi.org/10.1590/1413-81232015219.18922016 
30. Pinafo E, Carvalho BG, Nunes, EFPA. Descentralização da gestão: caminho percorrido, nós críticos e perspectivas. Ciênc Saúde Colet 2016;21(5):1511-24. https://doi.org/10.1590/1413-81232015215.18942015

31. Silva FF, Silva LAP. O espaço urbano e a desigualdade social no município de Canto do Buriti-Piauí: contrastes entre zona periférica e região central. Revista de Geografia 2016;33(1):131-47. Disponível em: https://periodicos.ufpe.br/revistas/ revistageografia/article/view/229186/23577.

32. Leite LM, Magalhães MA. Desigualdades intraestaduais no Espírito Santo: uma abordagem espacial exploratória. Revista de Economia 2012;38(1):55-92.

33. Prefeitura Municipal de Brejetuba. Conheça Brejetuba [Internet]. [acessado em 10 dez 2019]. Disponível em: https://www. brejetuba.es.gov.br/pagina/ler/1044/conheca-brejetuba.

34. Prefeitura de Vitória. História de Vitória [Internet]. [acessado em 10 dez 2019]. Disponível em: https://www.vitoria.es.gov.br/ turista/historia-de-vitoria.

35. Santos LMP, Costa AM, Girardi SN. Programa Mais Médicos: uma ação efetiva para reduzir iniquidades em saúde. Ciênc Saúde Colet 2015;20(11):3547-52. https://doi.org/10.1590/1413-812320152011.07252015

36. Malta DC, Santos MAS, Stopa SR, Vieira JEB, Melo EA, Reis AAC. A cobertura da Estratégia de Saúde da Família (ESF) no Brasil, segundo a Pesquisa Nacional de Saúde, 2013. Ciênc Saúde Colet 2016;21(2):327-38. https://doi.org/10.1590/141381232015212.23602015

37. Brasil. Ministério da Saúde. Secretaria de Gestão do Trabalho e da Educação na Saúde. Portaria n 30 , de 12 de fevereiro de 2014. Dispõe sobre o cumprimento das obrigações de oferta de moradia, deslocamento, alimentação e água potável pelo Distrito Federal e Municípios aos médicos participantes do Projeto Mais Médicos para o Brasil, nos termos da Portaria Interministerial $n^{\circ}$ 1.369/MS/MEC, de 8 de julho de 2013. Brasília: Diário Oficial da União; 2014. Disponível em: https:// bvsms.saude.gov.br/bvs/saudelegis/sgtes/2014/prt0030_12_02_2014.html\#: :text=Disp\%C3\%B5e\%20sobre\%20o\%20 cumprimento\%20das,8\%20de\%20julho\%20de\%202013.

38. Organização Pan-Americana da Saúde. Atenção à saúde em municípios de pequeno porte do Maranhão. Efeitos do Programa Mais Médicos [Internet]. Brasília: Organização Pan-Americana da Saúde; 2016 [acessado em 22 abr. 2020 ]. Disponível em: http://maismedicos.bvsalud.org/wp-content/uploads/2016/12/Estudo-MM-MA1-versa\%CC\%83oWEB.pdf

39. Santos MLM, Bertussi DC, Kodjaoglanian VL, Merhy EE. O que pode uma política? Problematizando a implementação do Programa Mais Médicos a partir da experiência de uma cidade brasileira. Interface 2019;23:e190052. https://doi.org/10.1590/ Interface.190052

40. Silva SP, Leite LM. Transbordamentos de pobreza e desigualdade em Minas Gerais: uma análise espacial considerando o efeito da fronteira interestadual. Rev Econ NE 2017;48(3):55-76.

41. Miranda GMD, Mendes ACG, Silva ALA, Santos Neto PM. A ampliação das equipes de saúde da família e o Programa Mais Médicos nos municípios brasileiros. Trab Educ Saúde 2017;15(1):131-45. https://doi.org/10.1590/1981-7746-sol00051

42. Giovanella L, Mendonça MHM, Fausto MCR, Almeida PF, Bousquat A, Lima JG, et al. A provisão emergencial de médicos pelo Programa Mais Médicos e a qualidade da estrutura das unidades básicas de saúde. Ciênc Saúde Colet 2016;21(9):2697708. https://doi.org/10.1590/1413-81232015219.16052016

43. Silva $H$, Baia $P$. Associação político-partidária e influência da estrutura de incentivos na adesão dos municípios às políticas de saúde: evidências do Projeto Mais Médicos para o Brasil. Saúde Soc 2018;27(2):615-31. https://doi.org/10.1590/S010412902018170494

44. Giovanella L, Mendonça MHM, Almeida PF, Escorel S, Senna MCM, Fausto MCR, et al. Saúde da família: limites e possibilidades para uma abordagem integral de atenção primária à saúde no Brasil. Ciênc Saúde Colet 2009;14(3):783-94. https://doi.org/10.1590/S1413-81232009000300014

45. Donabedian A. The quality of care. How can it be assessed? JAMA 1988;260(12):1743-8. https://doi.org/10.1001/ jama.260.12.1743

46. Mendes A, Carnut L, Guerra LDS. Reflexões acerca do financiamento federal da Atenção Básica no Sistema Único de Saúde. Saúde Debate 2018;(spe 1):224-243. https://doi.org/10.1590/0103-11042018S115

47. Schenkel MA. O papel da política de saúde sobre as desigualdades nas regiões do Brasil. Guaju 2017;3(2):104-21.

48. Rawls J. Justiça como eqüidade: uma concepção política, não metafísica. Lua Nova 1992;25:25-59. https://doi.org/10.1590/ S0102-64451992000100003

49. Vieira-da-Silva LM, Almeida Filho N. Eqüidade em saúde: uma análise crítica de conceitos. Cad Saúde Pública 2009;25(Supl 2):S217-S226.

50. Brasil. Ministério da Saúde. Ministério da Saúde. Gabinete do Ministro. Portaria Interministerial $n^{\circ} 1.369$, de 8 de julho de 2013. Dispõe sobre a implementação do Projeto Mais Médicos para o Brasil. Brasília: Diário Oficial da União; 2013. Disponível em: https://bvsms.saude.gov.br/bvs/saudelegis/gm/2013/pri1369_08_07_2013.html

51. Organização Pan-Americana da Saúde. Programa Mais Médicos no município do Rio de Janeiro. Mais acesso, equidade e resolutividade na APS. Brasília: Organização Pan-Americana da Saúde; 2016. Disponível em: https://iris.paho.org/bitstream/ handle/10665.2/34285/9788579671166-por.pdf?sequence=1\&isAllowed=y

52. Brasil. Presidência da República. Secretaria-Geral. Subchefia para Assuntos Jurídicos. Lei $n^{\circ} 13.958$, de 18 de dezembro de 2019. Institui o Programa Médicos pelo Brasil, no âmbito da atenção primária à saúde no Sistema Único de Saúde (SUS), e autoriza o Poder Executivo federal a instituir serviço social autônomo denominado Agência para o Desenvolvimento da Atenção Primária à Saúde (Adaps). Brasília: Diário Oficial da União; 2019. Disponível em: http://www.planalto.gov.br/ ccivil_03/_ato2019-2022/2019/lei/L13958.htm 